\title{
SOME PROBLEMS RELATING TO SOLAR LINE \\ IDENTIFICATION
}

\author{
A. H. GABRIEL \\ Astrophysics Research Unit, Culham Laboratory, Abingdon, Berkshire, England
}

\begin{abstract}
A review is given of some of the problems of classification and intensity analysis involving unidentified or recently identified lines in the ultraviolet solar spectrum. In particular, attention is drawn to the present position regarding two and three electron spectra in the soft X-ray region, and forbidden transitions within the ground configurations at longer wavelengths.
\end{abstract}

\section{Introduction}

Identification of observed spectra is normally a first step towards interpretation of the measured intensities of the lines. In addition to any contribution to fundamental atomic physics, such interpretation can yield information on the temperature/density structure of the solar atmosphere, as well as on the abundances of elements, dynamic processes and heating mechanisms.

Most of the more intense lines observed in the solar spectrum between $1.5 \AA$ and $2000 \AA$ have now been identified. In spite of this, there are a number of interesting problems surrounding the unidentified or recently identified lines. When the excitation mechanism departs significantly from LTE, as it does throughout the chromosphere and corona, it is of particular value to study those lines whose intensities are determined by more complex factors. These include forbidden and intersystem lines as well as transitions from autoionizing levels. The identification of such lines is therefore of considerable importance.

This paper will not attempt to review all the outstanding identification problems, but rather to select a few areas of particular interest involving unidentified or recently identified spectra. In many cases, these are illustrated by drawing on current work at the Astrophysics Research Unit. It is convenient to subdivide the lines considered in terms of the quantum numbers of the jumping electron.

\section{Transitions $2 \rightarrow 1$}

These transitions occur in hydrogen-like and helium-like ions, as well as through inner-shell transitions in lower stages of ionization. Recent problems are illustrated by the schematic spectra in Figure 1. This shows the appearance of spectra in the region of the helium-like O VII lines as observed in (a) laboratory sources, sparks, etc. and (b) from the Sun. On the long-wavelength side of the resonance line, $1 s^{2}{ }^{1} S$ $1 s 2 p^{1} P$, and intercombination line, $1 s^{2}{ }^{1} S-1 s 2 p{ }^{3} P$, additional lines are seen. In laboratory plasmas these appear as several lines of low intensity; in the Sun as a single intense line. At first, both these groups of features were thought to be of similar 
Laboratory plasma

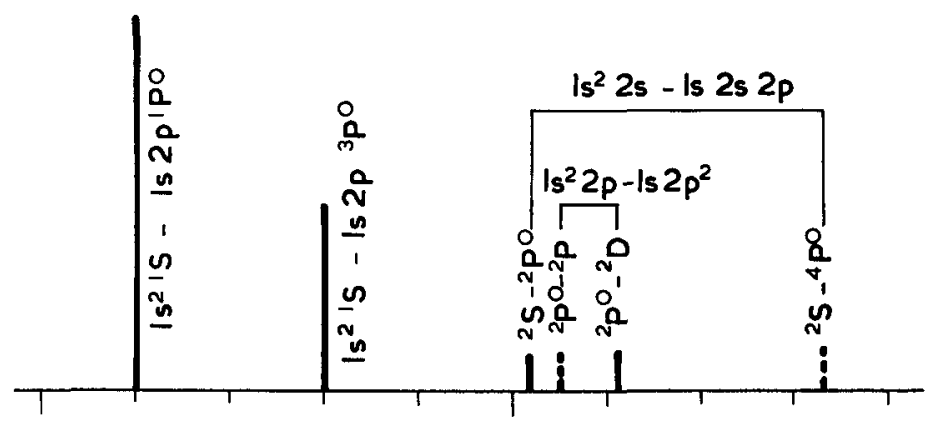

Solar corona

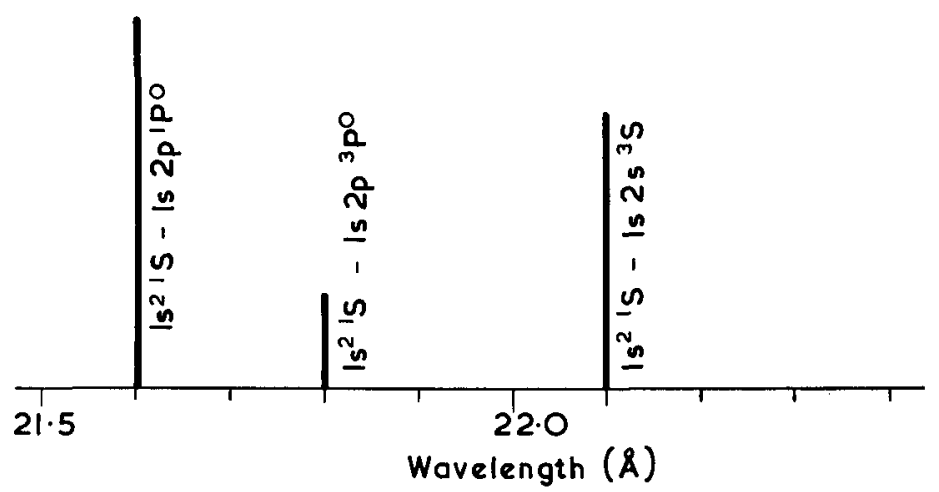

Fig. 1. Schematic representation of spectra observed in the vicinity of the OvI resonance line.

origin. However, the lines have now been classified (Gabriel and Jordan, 1969a) and shown to arise from different types of transitions.

\subsection{Dielectronic satellites}

The lines observed in laboratory plasmas are due to inner-shell transitions in lithiumlike ions, of the type $1 s^{2} 2 s-1 s 2 s 2 p$ or $1 s^{2} 2 p-1 s 2 p^{2}$. In all but very high density plasmas, the initial states are autoionizing levels, and are formed from the heliumlike ion by dielectronic recombination. Their intensity relative to the helium-like resonance line is independent of electron density $N_{e}$ but scales with the electron temperature as $T_{e}^{-1}$ and with the ionic charge as $Z^{4}$ (if $T_{e}$ is assumed to scale as $Z^{2}$ ). In typical conditions, this relative intensity might be approximately 0.01 for oxygen, 0.1 for silicon, 0.3 for calcium and 1 for iron. Such lines would not therefore be observed from oxygen in the solar spectra where the recording sensitivity for weak lines has so far been much less than in laboratory experiments but they would be expected from heavier elements, and have been reported (Neupert and Swartz, 1970) in iron in roughly the ratios predicted by theory. The present theory (Gabriel et al., 1969) should be valid up to oxygen and has been confirmed by laboratory experiments. For 
higher ions, a more complete theory will be required. The relative intensities will then serve to measure $T_{e}$ in active regions.

\subsection{HeLIUM-LIKE FORBIDDEN LINES}

The intense solar line in Figure 1 is due to the $1 s^{2}{ }^{1} S-1 s 2 s^{3} S$ forbidden line in OvII. The transition has also been seen in other ions from $\mathrm{CV}$ up to $\mathrm{CaXIX}$ and possibly Fexxv. The classification of these lines in the solar spectrum has shown that the $1 s 2 s{ }^{3} S$ level decays primarily by single-photon magnetic dipole emission and not by two-photon emission as previously expected. Following this, the transition probabilities calculated by Griem (1969) have been used to derive a theory for the relative intensities as a function of $N_{e}$ (Gabriel and Jordan, 1969b). It has now become clear that there is an error in the $Z$ scaling of Griem's transition probabilities, and the theory has been recalculated with semi-empirical values for the forbidden line transition probability (Freeman et al., 1970; Gabriel and Jordan, 1970). Recently, more precise values have been calculated by Drake (1971), and these are in satisfactory agreement with the semi-empirical values used by Freeman et al.

The forbidden line is comparable in intensity with the resonance and intercombination lines and to first order the ratios do not scale with $T_{e}$ or $Z$. However, above some critical density $N_{e}^{*}$, intensity is transferred from the forbidden to the intercombination line with increasing $N_{e}$, resulting in the extinction of the forbidden line at laboratory plasma densities. The critical density $N_{e}^{*}$ scales approximately as $Z^{13}$. We thus have a means of measuring $N_{e}$ over certain density ranges which can occur in solar active regions.

For heavier ions it is possible to see in the Sun both the dielectronic satellites and the forbidden line. A good example in silicon is shown in a recent spectrum by Walker and Rugge (1971), reproduced in Figure 2. These authors have identified many of the dielectronic satellites, including some in the two-electron configurations.

\section{Transitions $2 p \rightarrow 2 s$}

A number of recent identifications relating both to these transitions and to those of Section 4 have been made from solar spectra by Burton and Ridgeley (1970) and Freeman and Jones (1970) and from laboratory studies (Fawcett et al., 1970; Fawcett, 1970a, b).

$2 p \rightarrow 2 s$ transitions can occur in ions with ground configurations $1 s^{2} 2 s^{2} 2 p^{n}$ (n=0 to 5 ) or $1 s^{2} 2 s$. Allowed transitions of this type in lithium-like and beryllium-like ions are important through their application to the measurement of electron temperatures (Heroux, 1964). Many of these transitions have been measured recently in laboratory sources from sodium to chlorine and several have also been identified in the solar spectrum.

Intersystem lines are of particular value in solar analysis. Since both collisions and radiation compete in depopulating the upper levels, intensities relative to allowed lines will, over certain density ranges, depend on the density. In addition the low 


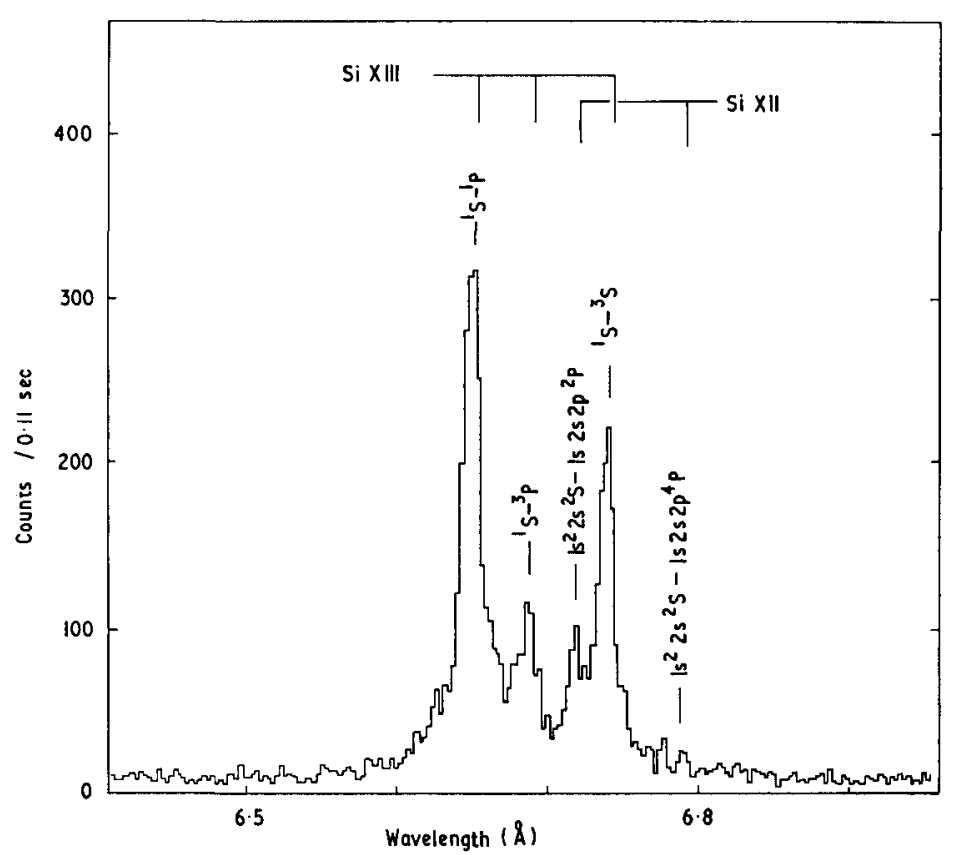

Fig. 2. Solar silicon lines observed in the X-ray region by Walker and Rugge (1971).

$f$-values makes it entirely safe to assume that such lines are optically thin in emission over a wide range of conditions. Because of this effect, such lines show a large limb brightening and are most readily observed in solar limb spectra. Using this technique, Burton and Ridgeley identify lines from CIII, NIII and IV, OI, III, IV and V and SiXI.

\section{Transitions $3 p \rightarrow 3 s$}

These will be the lowest transitions in ions with configurations from $3 s$ to $3 s^{2} 3 p^{5}$. A large number of lines are possible and it is thought that many of the solar lines in the region $200 \AA$ to $400 \AA$ are due to such transitions in iron. Recent laboratory work on elements between calcium and iron by Fawcett $(1970 \mathrm{~b})$ has led to classifications of some of the solar lines and has predicted others.

Intersystem lines in these configurations are not well known, only SiI to Si III being identified in the solar spectrum. Further work in this area could be important.

\section{Transitions $3 d \rightarrow 3 p$}

These transitions in configurations $3 s^{2} 3 p^{6} 3 d$ to $3 s^{2} 3 p$ in iron are responsible for the group of very intense lines observed in the solar spectrum between $170 \AA$ and $250 \AA$. The majority of these are now classified (Gabriel et al., 1966; Fawcett et al., 1967) although a few of the weakest lines remain unidentified. 


\section{Transitions $3 \rightarrow 2$}

Such transitions can take place in any ions with outer shell $n=2$ electrons. An outstanding problem occurs in the solar spectrum between $9 \AA$ and $18 \AA$. During solar flares, Neupert et al. (1967) in their OSO-III experiment observed greatly enhanced emission from a number of lines throughout this region. These they have tentatively classified, by comparison with calculated spectra, as $2 p-3 s$ and $2 p-3 d$ transitions in Fexvil to Fexxiv. With their wavelength resolution limited to $0.05 \AA$, they are only able to assign transition arrays and not individual terms. Their result is shown in Figure 3. Several of these lines have been produced in the spectrum of a low inductance spark by Feldman and Cohen (1967), but here again the resolution is insufficient to assign term values. Fawcett (1970a) has classified many of these transitions in the isoelectronic sequences from sodium to calcium. His extrapolations confirm Neupert's identifications and indicate probable dominant terms.

The changes in intensity of the solar flare lines as a function of time contain important information on the nature of the flare process. A more complete study of these lines at higher resolution is therefore indicated both in the Sun and in laboratory sources.

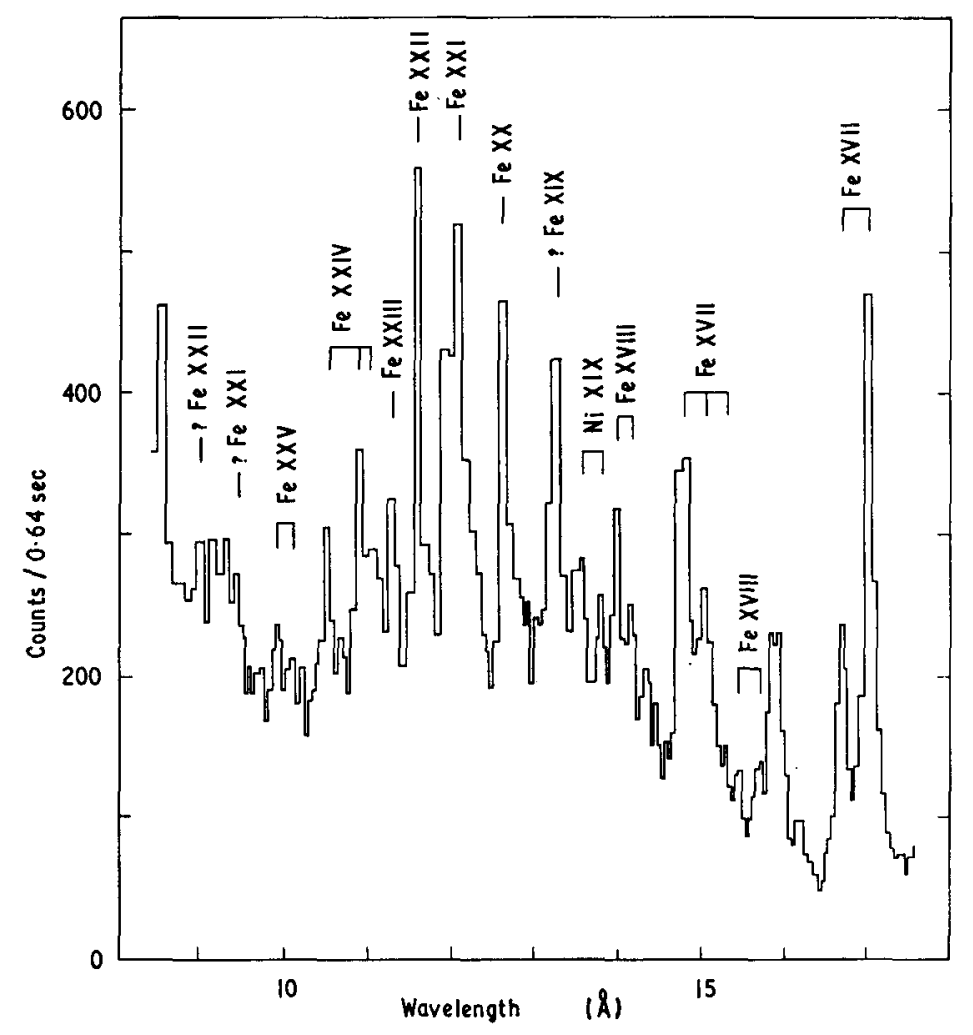

Fig. 3. Iron lines recorded during a solar flare from the OSO-III satellite (Neupert et al., 1967). 


\section{Transitions Within the Ground Configuration}

These transitions occur between terms or levels within the configurations $3 p^{n}$ or $2 p^{n}$ with $n=2$ to 4 . They are the forbidden lines which for coronal ions occur at wavelengths from $1000 \AA$ through to the infra-red. Many of those at wavelengths longer than $3000 \AA$ have now been classified, although some of these are tentative and several remain unidentified (Jefferies, 1969). Others lie in the ultraviolet and have only recently become accessible.

One approach to this problem is a detailed study of the shorter wavelength allowed transitions which connect to the ground configuration. Unfortunately a high wavelength accuracy is necessary here to give a moderate accuracy in prediction of the forbidden lines. However, the laboratory experiments described in Section 3 and 4 are now making some contribution to this problem. As an example, the recent classification by Fawcett of the FexiII multiplet $3 s^{2} 3 p^{2}{ }^{3} P-3 s 3 p^{3}{ }^{3} S$ gives the splitting of the ground ${ }^{3} P$ term. This provides the first laboratory confirmation of the forbidden solar ${ }^{3} P_{0}-{ }^{3} P_{1}$ and ${ }^{3} P_{1}-{ }^{3} P_{2}$ lines originally classified by Edlén (1942) by isoelectronic extrapolation.

The solar ultraviolet forbidden lines require the observation of isolated regions above the limb, using spectrographs carried in space vehicles. The solar limb spectra of Burton et al. (1967) enabled FeXI and XII lines to be identified in the region $1200 \AA-$ $1500 \AA$. This work can be greatly extended by measurements carried out recently during the total eclipse of $7 \mathrm{March}, 1970$. In a collaborative experiment between Imperial College London, the Astrophysics Research Unit, Harvard College Observatory and York University Toronto, a series of flash spectra were obtained from a rocket in the region $850 \AA$ to $2100 \AA$ at times throughout second contact (Speer et al., 1970). A frame from this data recorded close to totality, is shown in Figure 4 . The chromospheric spectrum has been obscured at this stage, except for prominences, and coronal lines appear as complete rings. Some lines from very high temperature regions show incomplete rings. In Figure 4, the allowed transitions having coronal extensions are indicated. All the other ring images are due to coronal forbidden lines, mainly from the configurations indicated above. Assignments include $3 p^{n}$ configurations in iron and nickel, and $2 p^{n}$ configurations in silicon and sulphur (Gabriel et al., 1971). Confirmation of these is difficult in some cases, and may be assisted by observation of the region $2000 \AA-3000 \AA$, planned for later eclipse flights.

\section{The Photospheric Spectrum}

This spectrum, which consists principally of absorption lines of neutrals and first ions of the transition elements, is now being extended further into the ultraviolet. The detail is complex and high wavelength resolution is necessary. The earlier work of Tousey (1964) carried these measurements down to $2200 \AA$. This has now been extended using a rocket-borne echelle instrument designed at Culham. In its first flight (Boland et al., 1971) it obtained a photospheric spectrum down to $2000 \AA$ which 

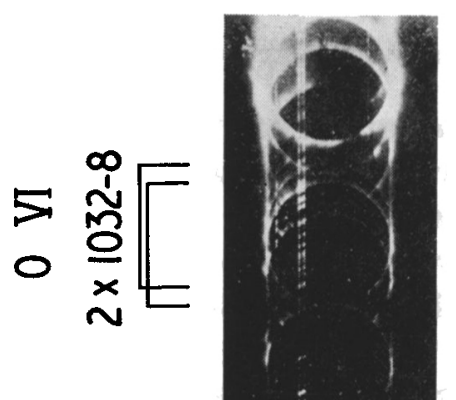

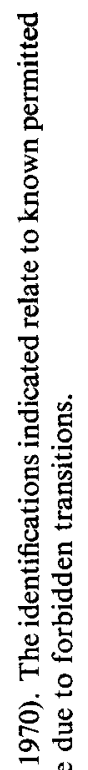
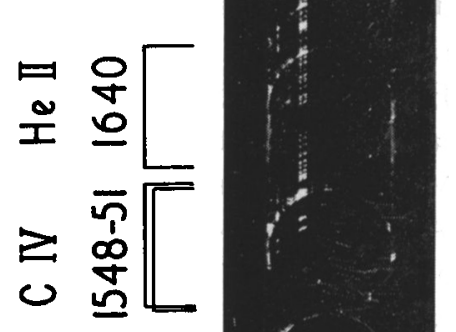

ปั

¿

害

造

옹.률

要

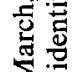

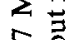

ㄴㅇㅇ

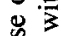

总密

$\stackrel{乛}{\Xi}$

昜

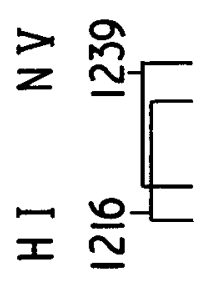

I

$\begin{array}{ll}\infty & \infty \\ 0 & \sim \\ 0\end{array}$

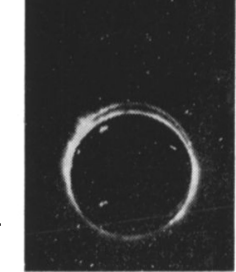

चे

تृ

放

E

昰

프

융

号

$\dot{+}$

in 
resulted in the identification of 663 new solar lines in the range $2000 \AA$ to $2200 \AA$. This spectrum is shown in Figure 5, in the familiar raster pattern resulting from crossed high and low dispersion. A more recent flight of this experiment has resulted in an extension of this spectrum to $1900 \AA$, and is at present being analysed.

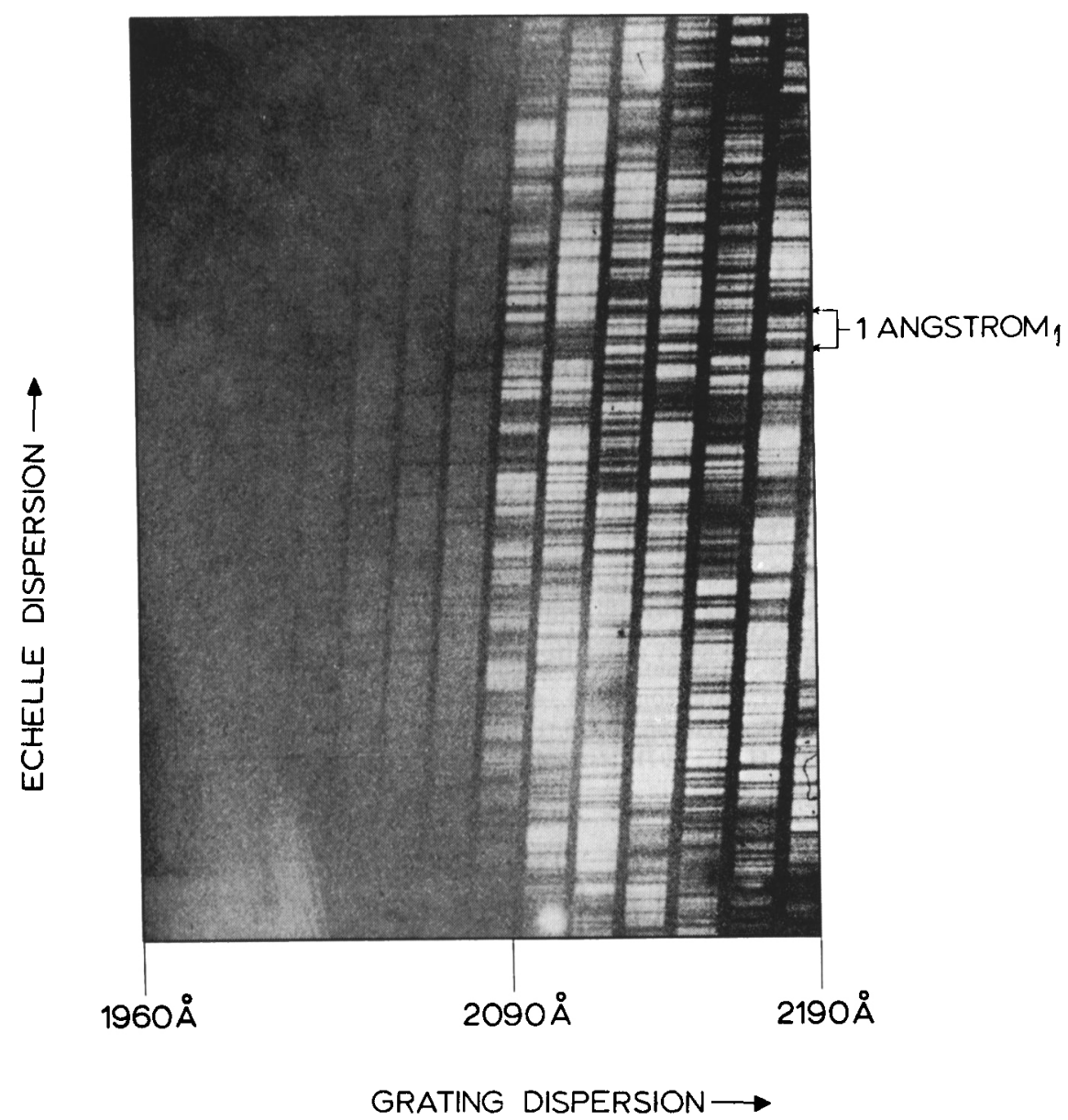

Fig. 5. Photospheric spectrum recorded at high spectral resolution (Boland et al., 1971).

\section{Acknowledgements}

I would like to thank those colleagues at the Astrophysics Research Unit and elsewhere, whose unpublished work has been used in this review. I am also grateful to Drs A. B. C. Walker and H. R. Rugge of the Aerospace Corporation for permission to quote their results in advance of publication. 


\section{References}

Boland, B. C., Jones, B. B., Wilson, R., Engstrom, S. F. T., and Noci, G.: 1971, Phil. Trans. Roy. Soc. A. $270,29$.

Burton, W. M. and Ridgeley, A.: 1970, Solar Phys. 14, 3.

Burton, W. M., Ridgeley, A., and Wilson, R.: 1967, Monthly Notices Roy. Astron. Soc. 135, 207.

Drake, G. W. F.: 1971, Phys. Rev. A. (in press).

Edlén, B.: 1942, $Z$. Astrophys. 22, 30.

Fawcett, B. C.: 1970a, J. Phys. B. [2], 3, 1152.

Fawcett, B. C.: 1970b, J. Phys. B. [2] 3, 1732.

Fawcett, B. C., Gabriel, A. H., and Saunders, P. A. H.: 1967, Proc. Phys. Soc. 90, 863.

Fawcett, B. C., Hardcastle, R. A., and Tondello, G.: 1970, J. Phys. B. [2], 3, 564.

Feldman, U. and Cohen, L.: 1968, Astrophys. J. 151, L55.

Freeman, F. F. and Jones, B. B.: 1970, Solar Phys. 15, 288.

Freeman, F. F., Gabriel, A. H., Jones, B. B., and Jordan, C.: 1971, Phil. Trans. Roy. Soc. A. $270,127$.

Gabriel, A. H. and Jordan, C.: 1969a, Nature 221, 947.

Gabriel, A. H. and Jordan, C.: 1969b, Monthly Notices Roy Astron. Soc. 145, 241.

Gabriel, A H. and Jordan, C.: 1970, Phys. Letters 32A, 166.

Gabriel, A. H., Fawcett, B. C., and Jordan, C.: 1966, Proc. Phys. Soc. 87, 825.

Gabriel, A. H., Jordan, C., and Paget, T. M.: 1969, Proc. 6th. Int. Conf. on Phys. of Electronic and Atomic Collisions, MIT Press, p. 558.

Gabriel, A. H., Garton, W. R. S., Goldberg, L., Jones, T. J. L., Jordan, C., Morgan, F. J., Nicholls, R. W., Parkinson, W. H., Paxton, H. J. B., Reeves, E. M., Shenton, D. B., Speer, R. J., and Wilson, R.: 1971, Astrophys. J. (in press).

Griem, H. R.: 1969, Astrophys. J. 156, L103.

Heroux, L.: 1964, Proc. Phys. Soc. 83, 121.

Jefferies, J. T.: 1969, Mem. Soc. Roy. Sci. Liege (5) 16, 213.

Neupert, W. M., Gates, W., Swartz, M., and Young, R.: 1967, Astrophys. J. 149, L79.

Neupert, W. M. and Swartz, M.: 1970, Astrophys. J. 160, L189.

Speer, R. J., Garton, W. R. S., Morgan, F. J., Nicholls, R. W., Goldberg, L., Parkinson, W. H., Reeves, E. M., Jones, T. J. L., Shenton, D. B., and Wilson, R.: 1970, Nature 226, 249.

Tousey, R.: 1964, Quart. J. Roy. Astron. Soc. 5, 123.

Walker, A. B. C. and Rugge, H. R.: 1971, Astrophys. J. (in press). 